\title{
Modulational instability and wave amplification in finite water depth
}

\author{
L. Fernandez ${ }^{1}$, M. Onorato ${ }^{2,3}$, J. Monbaliu ${ }^{1}$, and A. Toffoli ${ }^{4}$ \\ ${ }^{1}$ Department of Civil Engineering, KU Leuven, Kasteelpark Arenberg 40 box 2448, 3001 Heverlee, Belgium \\ ${ }^{2}$ Dipartimento di Fisica, Universita' di Torino, Via P. Giuria, Turin, 10125, Italy \\ ${ }^{3}$ INFN, Sezione di Torino, Via Pietro Giuria 1, 10125 Turin, Italy \\ ${ }^{4}$ Centre for Ocean Engineering Science and Technology, Swinburne University of Technology, P.O. Box 218, Hawthorn, \\ VIC., 3122, Australia
}

Correspondence to: A. Toffoli (toffoli.alessandro@gmail.com)

Received: 15 August 2013 - Published in Nat. Hazards Earth Syst. Sci. Discuss.: 2 October 2013

Revised: 19 February 2014 - Accepted: 20 February 2014 - Published: 27 March 2014

\begin{abstract}
The modulational instability of a uniform wave train to side band perturbations is one of the most plausible mechanisms for the generation of rogue waves in deep water. In a condition of finite water depth, however, the interaction with the sea floor generates a wave-induced current that subtracts energy from the wave field and consequently attenuates the instability mechanism. As a result, a plane wave remains stable under the influence of collinear side bands for relative depths $k h \leq 1.36$ (where $k$ is the wavenumber of the plane wave and $h$ is the water depth), but it can still destabilise due to oblique perturbations. Using direct numerical simulations of the Euler equations, it is here demonstrated that oblique side bands are capable of triggering modulational instability and eventually leading to the formation of rogue waves also for $k h \leq 1.36$. Results, nonetheless, indicate that modulational instability cannot sustain a substantial wave growth for $k h<0.8$.
\end{abstract}

\section{Introduction}

The occurrence of extreme waves (also known as freak or rogue waves) has an important role in many branches of physics and engineering (see, for example, Chabchoub et al., 2011; Onorato et al., 2013a, b; Chalikov, 2009; Babanin et al., 2011; Bitner-Gregersen and Toffoli, 2012; Toffoli et al., 2008b; Solli et al., 2007; Kibler et al., 2010; Bailung et al., 2011, among many others). Apart from a linear superposition of wave modes and the effect of currents on waves (caustic theory), the modulational instability of a plane wave to side band perturbations remains the most plausible mechanism for the formation of rogue waves in deep water (Zakharov and Ostrovsky, 2009; Osborne, 2010; Onorato et al., 2013b; Kharif et al., 2009), i.e. $k_{0} h \rightarrow \infty$, where $k_{0}$ is the wavenumber of the plane wave and $h$ is the water depth. Basically, this is a generalisation of the Benjamin and Feir (1967) or modulational instability (Zakharov, 1968) and can be described by the nonlinear Schrödinger (NLS) equation (Zakharov, 1968), which is derived from the Euler equations by assuming that waves are weakly nonlinear (i.e. the wave steepness $\varepsilon=k_{0} a_{0} \ll 1$, where $a_{0}$ is the amplitude of the carrier wave) and have narrow bandwidth $\left(\Delta k / k_{0} \ll 1\right.$, where $\Delta k$ is the modulation wavenumber). For a propagation in one dimension, a linear stability analysis of the NLS equation indicates that unstable disturbances can lead to an exponential growth of a small-amplitude modulation and hence to rogue waves (see e.g. Osborne, 2010).

If two-dimensional propagation is allowed, a $2+1$ form of the NLS equation indicates that unstable disturbances are not only limited to the ones propagating collinearly with the plane wave, but also include modes that propagate at an angle with respect to the carrier. In this regard, it is important to mention that the region of instability is stretched over a narrow domain, forming an angle of about $35.5^{\circ}$ with the mean wave direction towards high wavenumbers (see the instability diagram in Fig. 1 of Gramstad and Trulsen, 2011, for example). Although the most unstable modes remain collinear in water of infinite depth, oblique perturbations tend to dominate the modulational instability for conditions of arbitrary water depths when $k_{0} h<\varepsilon^{-1}$ (Trulsen and Dysthe, 1996). This is also confirmed by laboratory experiments in a relatively wide long wave flume (Trulsen et al., 1999), where 
a plane wave without any initial seeding of unstable modes was observed to transfer energy towards a lower oblique side band (see also Babanin et al., 2011; Ribal et al., 2013). Direct numerical simulations of the $2+1$ NLS equation, furthermore, substantiate that not only can oblique disturbances sustain modulational instability, but they are also capable of triggering the formation of rogue waves (Osborne et al., 2000; Slunyaev et al., 2002).

For conditions of more finite water depths, $k_{0} h \approx O(1)$, wave-induced mean flow gradually subtracts energy from the wave fields with a concurrent weakening of the modulational instability mechanism (see e.g. Slunyaev et al., 2002; Benjamin, 1967; Whitham, 1974; Janssen and Onorato, 2007; McLean, 1982; Benney and Roskes, 1969). As a result, there is a reduction in the region of instability (see Fig. 1 in Gramstad and Trulsen, 2011), leading to a complete stabilisation of collinear modes at a critical relative water depth $k_{0} h=1.36$ (Benjamin, 1967; Janssen and Onorato, 2007). Beyond this threshold, nevertheless, oblique perturbations still remain unstable and numerical simulations of the $2+1$ NLS equation, in this respect, confirm that such modes can still trigger very large amplitude waves (Slunyaev et al., 2002; Didenkulova et al., 2013). Furthermore, direct numerical simulations of the Euler equation also indicate that directional components can sustain the formation of extreme waves in random directional wave fields in water of finite depth, leading to substantial deviations from standard second-order based statistics (Toffoli et al., 2009).

A systematic analysis of the effect of oblique perturbations on the nonlinear dynamics of a plane wave has not been attempted yet and hence the transition between infinite and finite depths still remains not completely clear. It is reasonable to expect, moreover, that the region of instability would eventually vanish for sufficiently shallow water depths. Therefore, there should exist a lower limit beyond which wave amplitude growth would cease. In the present paper, the nonlinear evolution of a plane wave in relative water depth gradually varying from deep water $\left(k_{0} h \rightarrow \infty\right)$ to shallow water $\left(k_{0} h \rightarrow 0\right)$ conditions and for different degrees of nonlinearity (i.e. wave steepness) is discussed. The problem is approached numerically by solving the Euler equations for the wave motion with a higher order spectral method (HSOM) (West et al., 1987; Dommermuth and Yue, 1987). In the next two sections, a brief description of the model and its initialisation is presented. In Sect. 4, the temporal evolution of the wave field is discussed; nonlinear energy transfer from the carrier to the unstable side bands and wave amplitude growth are presented. In the final section, some concluding remarks are given.

\section{The model}

Assuming an irrotational, inviscid and incompressible fluid flow, a velocity potential $\phi(x, y, z, t)$ that satisfies the
Laplace equation everywhere in the fluid can be defined. For the present study, a constant water depth is also assumed. At the bottom $(z=-h)$ the boundary condition is such that the vertical velocity is zero (i.e. $\left.\phi_{z}\right|_{h}=0$ ). At the free surface $z=$ $\eta(x, y, t)$, the kinematic and dynamic boundary conditions are satisfied for the free surface elevation and the velocity potential at the free surface $\psi(x, y, t)=\phi(x, y, \eta(x, y, t), t)$. Using the free surface variables these conditions can be written as follows (see e.g. Zakharov, 1968):

$$
\begin{aligned}
\psi_{t}+g \eta+\frac{1}{2}\left(\psi_{x}^{2}+\psi_{y}^{2}\right)-\frac{1}{2} W^{2}\left(1+\eta_{x}^{2}+\eta_{y}^{2}\right) & =0 \\
\eta_{t}+\psi_{x} \eta_{x}+\psi_{y} \eta_{y}-W\left(1+\eta_{x}^{2}+\eta_{y}^{2}\right) & =0
\end{aligned}
$$

where the subscripts indicate the partial derivatives, and $W(x, y, t)=\left.\phi_{z}\right|_{\eta}$ represents the vertical velocity at the free surface.

The temporal evolution of the surface elevation can be estimated directly from the system of Eqs. (1) and (2). To this end, a higher order spectral method (HOSM), which was independently proposed by West et al. (1987) and Dommermuth and Yue (1987), is applied. Note, however, that an interesting comparison between these two approaches in Clamond et al. (2006) reveals that the formulation in Dommermuth and Yue (1987) is less consistent than the one proposed by West et al. (1987). Therefore, only the latter is applied herein.

HOSM is based on a pseudo-spectral approach that uses a series expansion in the wave steepness $\varepsilon$ of the velocity potential of the form

$\phi(x, y, z, t)=\sum_{m=1}^{M} \phi^{(m)}(x, y, z, t)$,

where each $\phi^{(m)}$ is a quantity of order $O\left(\varepsilon^{m}\right)$. In Eq. (3), $M$ represent the order of nonlinearity that is considered. A Taylor expansion around $z=0$ is then performed for each $\phi^{(m)}$ term and combined with the above expansion for the potential. After collecting all terms at each order in wave steepness, we obtain the following system:

$$
\begin{aligned}
& \phi^{(1)}(x, y, z=0, t)=\psi(x, y, t) \\
& \phi^{(m)}(x, y, z=0, t)= \\
& \quad-\sum_{k=1}^{m-1} \frac{\eta^{k}}{k !} \frac{\partial^{k}}{\partial z^{k}} \phi^{(m-k)}(x, y, z=0, t)
\end{aligned}
$$

for $m=2,3, \ldots, M$. Following West et al. (1987), $W(x, y, t)$ can also be similarly expanded in series of terms of order $O\left(\varepsilon^{m}\right)$ :

$W(x, y, t)=\sum_{m=1}^{M} W^{(m)}(x, y, t)$, 


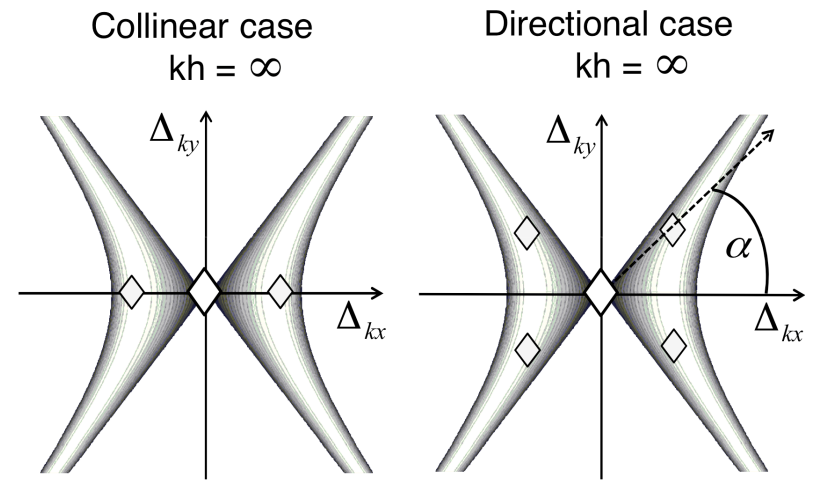

Fig. 1. Instability region and location of side bands for $k h \rightarrow$ $\infty$ : collinear disturbances (left panel); oblique disturbances (right panel).

where the terms $W^{(m)}$ are computed from the $\phi^{(m)}$ terms:

$W^{(m)}(x, y, t)=\sum_{k=0}^{m-1} \frac{\eta^{k}}{k !} \frac{\partial^{k+1}}{\partial z^{k+1}} \phi^{(m-k)}(x, y, z=0, t)$.

For the case of a rectangular domain in space with dimensions $L_{x}$ and $L_{y}$ in $x$ and $y$ and assuming periodicity in both directions for the wave field, we can use the following expression based on a double Fourier series for each $\phi^{(m)}$ term in finite water depth (see e.g. Dean and Dalrymple, 2000):

$\phi^{(m)}(x, y, z, t)=$

$\sum_{k, l} c_{k, l}^{(m)} \frac{\cosh \left[k_{k, l}(z+h)\right]}{\cosh \left(k_{k, l} h\right)} \cos \left(\omega t-\boldsymbol{k}_{k, l} \cdot \boldsymbol{x}\right)$,

with wavenumbers $k_{k, l}=\left|\boldsymbol{k}_{k, l}\right|$ and $\boldsymbol{k}_{k, l}=\left(k_{x}, k_{y}\right)=$ $\left(\frac{2 \pi k}{L_{x}}, \frac{2 \pi l}{L_{y}}\right) ; \omega=\sqrt{g\left|\boldsymbol{k}_{k, l}\right|}$ is the angular frequency. The time-dependent modal coefficients $c_{k, l}^{(m)}(t)$ of the potentials $\phi^{(m)}$ can be computed from Eq. (4) by using a twodimensional (fast) Fourier transform when the free surface elevation and the free surface velocity potentials are given as input.

Here, we considered both a third- and fifth-order expansion (i.e. $M=3$ and 5). The former allows the inclusion of four wave interactions (see Tanaka, 2001a, b), which is directly responsible for modulational instability (class-I instability). The latter also includes higher order interactions, which are responsible for class-II instability and concurrently for crescent waves (see, for example, McLean, 1982; Kristiansen et al., 2005; Francius and Kharif, 2006).

After evaluating the vertical velocity at the free surface at order $M$, the free surface velocity potential $\psi(x, y, t)$ and the surface elevation $\eta(x, y, t)$ can be integrated in time from Eqs. (1) and (2). The time integration is then performed by means of a fourth-order Runge-Kutta method with a constant time step. All aliasing errors generated in the nonlinear terms are removed (see West et al., 1987; Tanaka, 2001b,
Collinear case
$\mathrm{kh}=1.36$

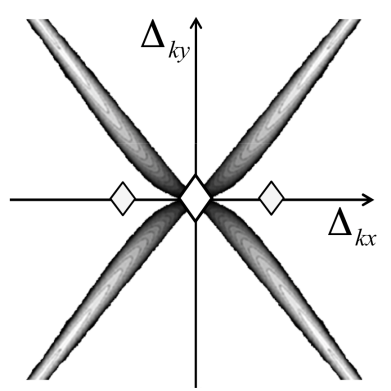

Directional case

$\mathrm{kh}=1.36$

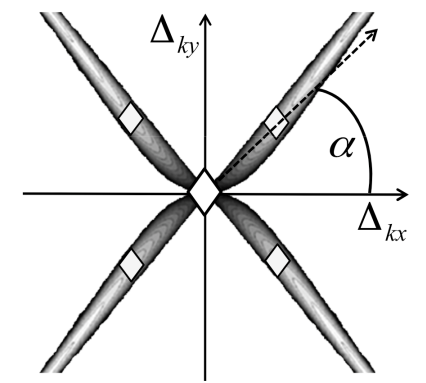

Fig. 2. Instability region and location of side bands for $k h=$ 1.36: collinear disturbances (left panel); oblique disturbances (right panel).

for details). Note, however, that no additional terms were included to take into account wave dissipation.

A concise review of HOSM can be found in Tanaka (2001a), while applications of this method to the nonlinear dynamics of surface gravity waves can be found in e.g. Mori and Yasuda (2002), Ducrozet et al. (2007), Toffoli et al. (2008a, b, 2010a, b), and Xiao et al. (2013), among others. Note that other numerical methods have also been proposed by Annenkov and Shrira (2001) and Clamond and Grue (2001). A comparative analysis between the performance of the HOSM and other numerical approaches can be found in Clamond et al. (2006).

\section{Initial conditions}

The model simulates the temporal evolution of an initial surface $\eta(x, y, t=0)$ and the concurrent velocity potential $\psi(x, y, t=0)$ with periodic boundaries. For the present study, the input surface and potential were defined by superimposing a plane (carrier) wave and four infinitesimal (small-amplitude) unstable side band perturbations. For convenience, we defined the carrier as a monochromatic wave with wavelength $L_{0}=156 \mathrm{~m}$ (wave period $T_{0}=10 \mathrm{~s}$ in deep water) and propagating along the $x$ direction. Several values of wave amplitude were applied to vary the wave steepness and hence the degree of nonlinearity. Overall, wave fields with the following steepness were used: $k_{0} a_{0}=0.1$, 0.12 and 0.14 , where $a_{0}$ is the amplitude of the plane wave. Each configuration was then tested within a wide range of water depths, varying from infinite to finite conditions (i.e. $0.5<k_{0} h<\infty$ ).

The four small-amplitude perturbations were carefully selected within the unstable region of the instability diagram and with amplitude equivalent to $0.05 \%$, the one of the carrier wave. The modulational wavenumbers $\Delta K_{x}$ and $\Delta K_{y}$ were defined such that the wave packets contain five waves under the modulation along the $x$ directional of propagation. 

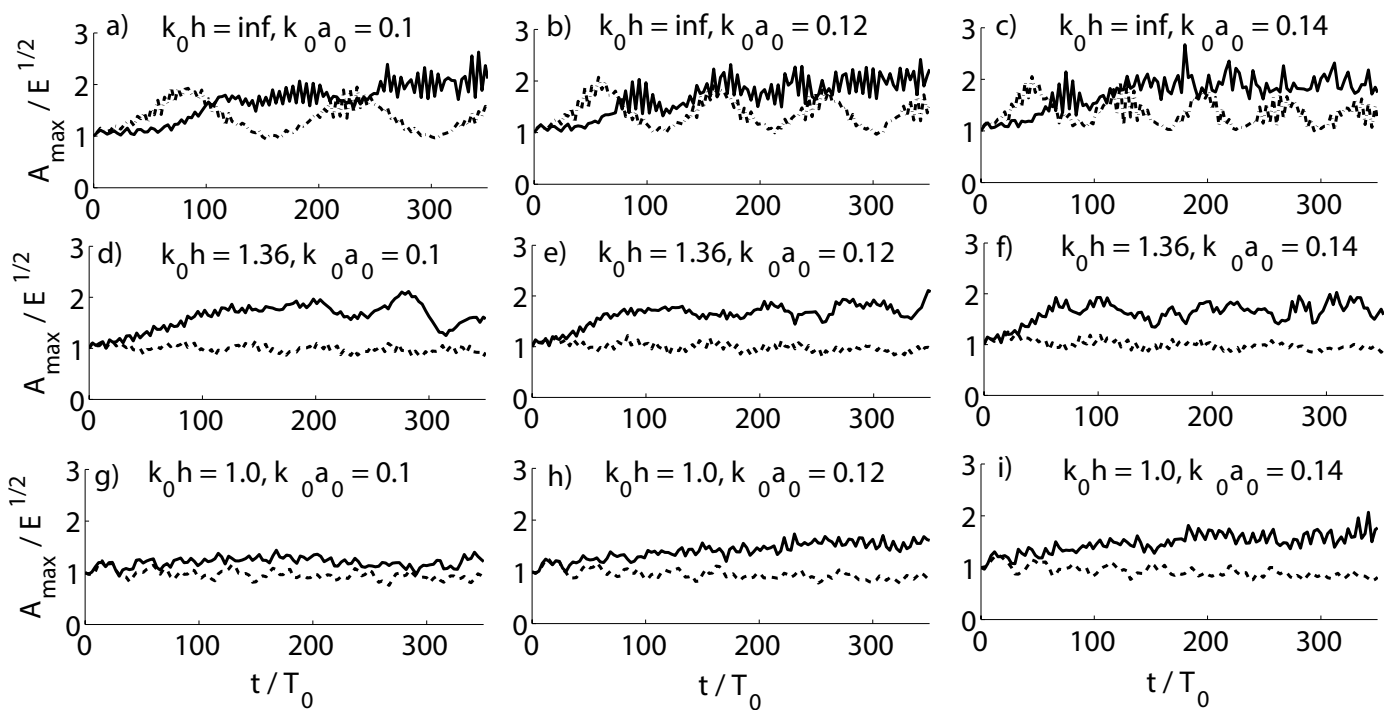

Fig. 3. Temporal evolution of the normalised maximum amplitude: plane wave seeded with collinear perturbations (dashed line); plane wave seeded with oblique perturbations (solid line). These results have been summarised in Figs. 4, 5 and 6 . (I.e: the maximum value of normalised maximum amplitudes shown in (c) $\left(k_{0}=\infty\right.$ and $\left.k_{0} a_{0}=0.14\right)$, for oblique perturbations (solid line), corresponds to the given value of $k h=$ inf in Fig. 6 for the oblique case.)

Overall, two lower (i.e. $\left[-\Delta K_{x}, \Delta K_{y}\right],\left[-\Delta K_{x},-\Delta K_{y}\right]$ ) and two upper (i.e. $\left[\Delta K_{x}, \Delta K_{y}\right],\left[-\Delta K_{x},-\Delta K_{y}\right]$ ) unstable modes were defined with $\Delta K_{y} / \Delta K_{x} \approx 0.7$ for $k h>1.36$, $\Delta K_{x} / K_{0} \approx 0.20$ and $\Delta K_{y} / K_{0} \approx 0.14$. A schematic representation showing the instability diagram and the location of the selected modes is presented in Figs. 1 and 2 for $k h \rightarrow \infty$ and $k h=1.36$, respectively.

The effect of collinear perturbations was also investigated by imposing $\Delta K_{y}=0$ (see left panels in Figs. 1 and 2). Note, however, that the resulting lower and upper collinear perturbations have an amplitude that is equivalent to $0.1 \%$ of the carrier, i.e. twice the amplitude of an oblique side band.

The dimension of the physical domain was defined by a mesh of $256 \times 256$ points. The resolution in both dimensions was $\Delta x=\Delta y=6.24 m$ so that the domain includes 10 wavelengths and hence a dominant wave is discretised by 25 grid points. The time step was chosen equal to $\Delta t=$ $T_{0} / 150=0.067 \mathrm{~s}$. On the whole, the simulations estimated the evolution of the surface and velocity potential over a time frame of 350 dominant periods.

\section{Temporal evolution of wave amplitude}

At each time step, the maximum value of the wave amplitude was estimated from the resulting output surface. A summary of the temporal evolution of the maximum amplitude as normalised by the standard deviation of the wave envelope $E^{1 / 2}$ is presented in Fig. 3 for different relative depths and steepness. For simplicity only results that were obtained by applying a fifth-order expansion (i.e. $M=5$ ) in the HOSM are presented in this figure.
As a consequence of wave-wave interactions, the initial configuration is expected to evolve in time, producing an energy transfer from the carrier wave to the unstable side bands, with the lower disturbances growing faster than the upper ones (see e.g. Lo and Mei, 1987; Tulin and Waseda, 1999). In a condition of deep water, this energy transfer is followed by a growth of the modulation that leads to a substantial increase of the wave amplitude. This amplification is triggered under the influence of both collinear and oblique disturbances. The process, however, seems to occur more rapidly under the influence of the former (see Fig. 3a, b and c). It is interesting to note, in this respect, that collinear disturbances also induce a recurrence in the phenomenon with a sequence of modulation and demodulation of the input surface (cf., for example, Ribal et al., 2013). When seeded with oblique side band perturbations, on the other hand, no significant evidence of recurrence can be detected. Despite some oscillations in the value of the amplitude, however, there is evidence for a robust monotonic growth of the wave amplitudes up to two times the initial value.

With the reduction in relative water depth, the region of collinear unstable modes gradually shrinks with a concurrent attenuation of the wave amplitude growth. Eventually, for the critical relative depth $k_{0} h=1.36$, collinear modulations become completely stable. As a consequence, energy transfer to collinear side bands no longer occurs and concurrently amplitude growth ceases, regardless of the value of steepness of the initial surfaces (see dashed line in Fig. 3d, e and $\mathrm{f}$ ). Oblique modulations, nevertheless, still remain unstable and grow at the expense of the plane wave. Note that first evidence of an energy transfer to oblique side bands can 


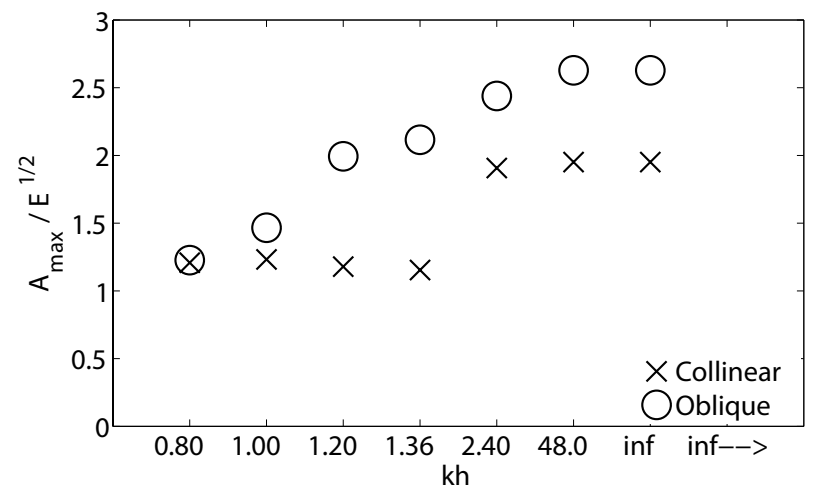

Fig. 4. Maximum wave amplification as a function of the relative water depth for $k_{0} a_{0}=0.1, M=5$.

be found in Trulsen et al. (1999), albeit for fairly deep water. In the physical space, the growth of oblique perturbations results in an amplification of the modulation, which roughly doubles its initial amplitude (see solid line in Fig. 3d, e and f). It is also worth mentioning that the timescale for this amplification remains comparable with the one observed in deep water, namely of the order of 100 wave periods. The process speeds up slightly with the increase in the degree of nonlinearity (wave steepness) though.

It is important to mention that the timescales are consistent with the generation of rogue waves in the laboratory (see, in this regard, a recent comparison between experimental data and HOSM simulations in Toffoli et al., 2013).

Despite the fact that the region of instability keeps compressing for further reductions of the relative water depth (see e.g. Gramstad and Trulsen, 2011), oblique unstable modes still sustain modulational instability and amplitude growth for $k_{0} h<1.36$. For the specific case of $k_{0} h=1$ (see Fig. 3g, $\mathrm{h}$ and $\mathrm{i}$ ), however, the effect on the modulation attenuates notably. For a low steepness $\left(k_{0} a_{0}=0.1\right.$ for this example), particularly, wave amplitude does not depart significantly from the input condition. An increase in steepness seems, however, to reactivate the mechanism, inducing a substantial wave amplification under the influence of oblique disturbances. It is remarkable, in this regard, that the modulation still doubles its initial amplitude for the largest value of steepness considered in this study (i.e. $k_{0} a_{0}=0.14$ ). This result is consistent with previous simulations of the $2+1$ NLS (Slunyaev et al., 2002), which demonstrated that rogue waves can still be generated in water of finite depth (i.e. $k_{0} h<1.36$ ), when a plane wave is seeded by appropriate oblique side bands.

In order to summarise the results of all simulations, the maximum wave amplitude (as normalised by $E^{1 / 2}$ ) is presented as a function of the relative water depth in Figs. 4, 5 and 6.

On the whole, it is interesting to note that collinear disturbances sustain a substantial amplification of an initially small amplitude modulation (up to twice the initial value) until

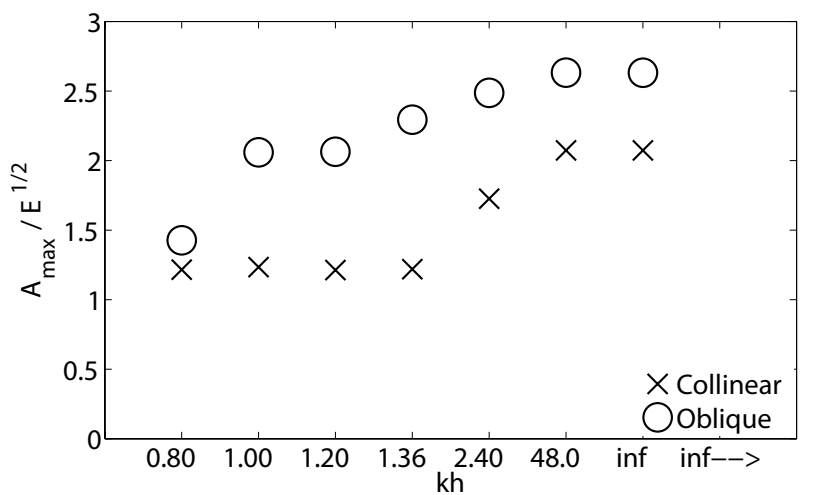

Fig. 5. Maximum wave amplification as a function of the relative water depth for $k_{0} a_{0}=0.12, M=5$.

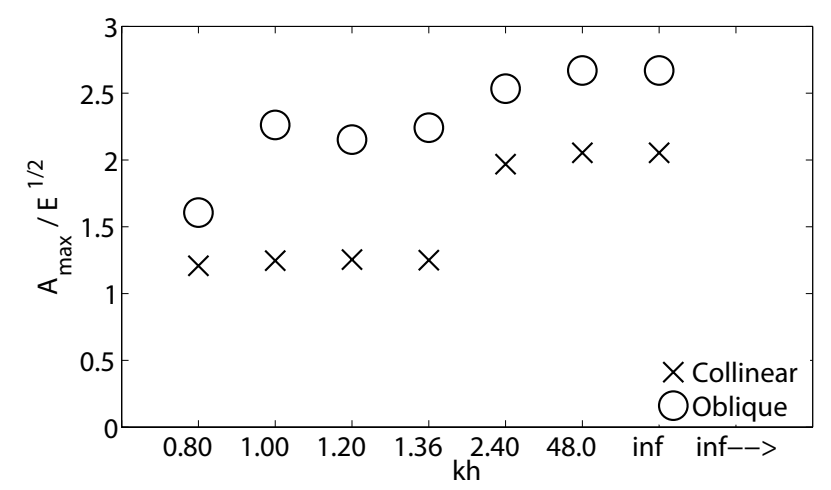

Fig. 6. Maximum wave amplification as a function of the relative water depth for $k_{0} a_{0}=0.14, M=5$.

relatively shallow water conditions with $k_{0} h$ as low as 2.4. For $k_{0} h \leq 1.36$, amplitude growth ceases completely under the influence of collinear side bands. Oblique perturbations, on the other hand, produce a substantially stronger amplification of the initial modulation already for deep water conditions. In contrast with the behaviour shown by a plane wave seeded with collinear disturbances, the degree of amplification reduces much more gradually, starting from $k_{0} h<48$. Nevertheless, a notable wave amplification still withstands also for $k_{0} h \leq 1.36$. It is worth mentioning, however, that the modulation does not grow significantly for relative water depth $k_{0} h \leq 0.8$.

We remark that results presented so far were obtained using $M=5$ in the HOSM and hence nonlinear mechanisms other than modulational instability (class-I) were included in the simulations. As class-II instability becomes dominant for initial wave trains of steepness as low as 0.12 (for example, Su and Green, 1985; Fructus et al., 2005), higher order nonlinear terms might, therefore, have played a role in the observed wave growth, especially for $k_{0} h=1$ (see Kristiansen et al., 2005; Francius and Kharif, 2006). In order to verify this conjecture, a comparison between simulations performed with $M=3$ and $M=5$ is presented in Fig. 7, for a 


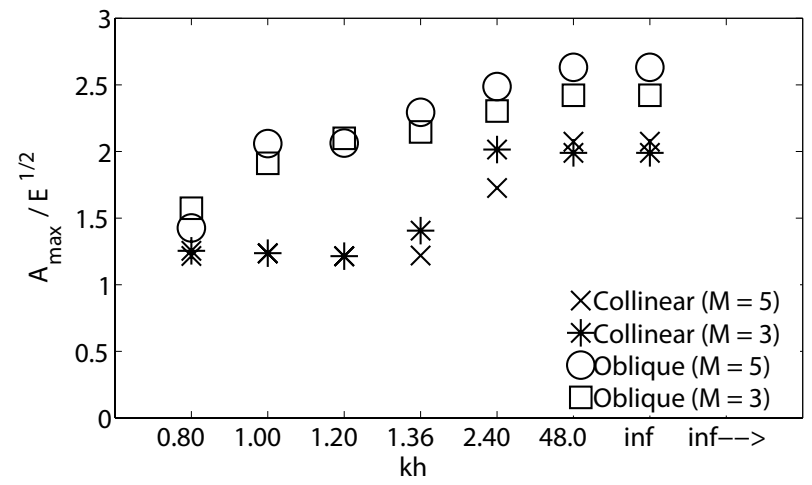

Fig. 7. Comparison with different orders.

wave steepness $k_{0} a=0.14$. Results seem to indicate, in this regard, that higher order terms (class-II instability) have in fact a notable effect on amplitude growth (at least for relative water depth as low as $k_{0} h=1$ ) when oblique perturbations are applied. There is no significant difference if collinear perturbations are used, though.

\section{Conclusions}

Nonlinear wave dynamics in finite water depth was investigated numerically by means of direct numerical simulations of the Euler equation solved with a higher order spectral method (West et al., 1987). Particularly, simulations were undertaken to investigate the role of oblique unstable perturbations in withstanding modulational instability beyond the critical relative water depth $k_{0} h=1.36$.

Simulations were carried out by tracking the temporal evolution of an initial surface composed by a plane wave and four oblique side band perturbations carefully selected within the region of instability. The physical domain was defined to include 10 dominant wavelengths and discretised with $256 \times 256$ grid points. A time step corresponding to $T_{0} / 150$, where $T_{0}$ is the period of the plane wave, was imposed. Runs were performed for different values of the steepness of the carrier wave and for different water depths so that a wide range of relative depths was defined here and ranged from deep to shallow water conditions $\left(0.5<k_{0} h<\infty\right)$. Configurations based on collinear perturbations were also applied for comparison.

As expected, results indicated that modulational instability ceases quite suddenly at $k_{0} h=1.36$, when the plane wave is seeded with collinear perturbations in agreement with Benjamin (1967) and Janssen and Onorato (2007). Under the influence of unstable side bands, however, modulational instability survives beyond this critical water depth and a significant amplification can be achieved also for relatively shallow water with $k_{0} h<1.36$, provided the perturbations are properly selected within the instability region. This is also in agreement with other experimental and numerical studies
(Yuen and Lake, 1982; Trulsen and Dysthe, 1996; Trulsen et al., 1999; Tulin and Waseda, 1999; Gramstad and Trulsen, 2011; Toffoli et al., 2013). For relative water depth $k_{0} h=$ 0.8 , simulations did not indicate any significant growing of the modulations, though.

Acknowledgements. F.W.O. project G.0333.09 and EU project EXTREME SEAS (contract SCP8-GA-2009-234175) are acknowledged. The authors also acknowledge the Hercules Foundation and the Flemish Government department EWI for providing access to the Flemish Supercomputer Center.

Edited by: E. Pelinovsky

Reviewed by: two anonymous referees

\section{References}

Annenkov, S. Y. and Shrira, V. I.: Numerical modeling of waterwave evolution based on the Zakharov equation, J. Fluid. Mech., 449, 341-371, 2001.

Babanin, A. V., Waseda, T., Kinoshita, T., and Toffoli, A.: Wave breaking in directional fields, J. Phys. Oceanogr., 41, 145-156, 2011.

Bailung, H., Sharma, S. K., and Nakamura, Y.: Observation of Peregrine Solitons in a Multicomponent Plasma with Negative Ions, Phys. Rev. Lett., 107, 255005, doi:10.1103/PhysRevLett.107.255005, 2011.

Benjamin, T. B.: Instability of periodic wave trains in nonlinear dispersive systems, Proc. Roy. Soc. London, A299, 59-75, 1967.

Benjamin, T. B. and Feir, J. E.: The disintegration of wave trains on deep water, Part I. Theory, J. Fluid Mech., 27, 417-430, 1967.

Benney, D. J. and Roskes, G. J.: Wave instabilities, Stud. Appl. Math, 48, 377-385, 1969.

Bitner-Gregersen, E. M. and Toffoli, A.: On the probability of occurrence of rogue waves, Nat. Hazards Earth Syst. Sci., 12, 751762, doi:10.5194/nhess-12-751-2012, 2012.

Chabchoub, A., Hoffmann, N., and Akhmediev, N.: Rogue wave observation in a water wave tank, Phys. Rev. Lett., 106, 204502, doi:10.1103/PhysRevLett.106.204502, 2011.

Chalikov, D.: Freak waves: Their occurrence and probability, Phys. Fluids, 21, 1-4, 2009.

Clamond, D. and Grue, J.: A fast method for fully nonlinear waterwave computations, J. Fluid Mech., 447, 337-355, 2001.

Clamond, D., Francius, M., Grue, J., and Kharif, C.: Long time interaction of envelope solitons and freak wave formations, Europ J. Mech. B/Fluids, 25, 536-553, 2006.

Dean, R. and Dalrymple, R.: Water Wave Mechanics for Engineers and Scientists, Advanced Series on Ocean Engineering - vol. 2, World Scientific Pub. Co., Singapore, reprint, 2000.

Didenkulova, I., Nikolkina, I., and Pelinovsky, E. N.: Rogue waves in the basin of intermediate depth and the possibility of their formation due to the modulational instability, JETP letters, 97, 194198, 2013.

Dommermuth, D. G. and Yue, D. K.: A high-order spectral method for the study of nonlinear gravity waves, J. Fluid Mech., 184, 267-288, 1987. 
Ducrozet, G., Bonnefoy, F., Le Touzé, D., and Ferrant, P.: 3-D HOS simulations of extreme waves in open seas, Nat. Hazards Earth Syst. Sci., 7, 109-122, doi:10.5194/nhess-7-109-2007, 2007.

Francius, M. and Kharif, C.: Three dimensional instabilities of periodic gravity waves in shallow water, J. Fluid Mech., 561, 417437, 2006.

Fructus, D., Kharif, C., Francius, M., Kristiansen, Ø., Clamond, D., and Grue, J.: Dynamics of crescent water wave patterns, J. Fluid Mech., 537, 155-186, 2005.

Gramstad, O. and Trulsen, K.: Hamiltonian form of the modified nonlinear Schrödinger equation for gravity waves on arbitrary depth, J. Fluid Mech., 670, 404-426, 2011.

Janssen, P. A. E. M. and Onorato, M.: The intermediate water depth limit of the Zakharov equation and consequences for wave prediction, J. Phys. Oceanogr., 37, 2389-2400, 2007.

Kharif, C., Pelinovsky, E., and Slunyaev, A.: Rogue Waves in the Ocean, Advances in Geophysical and Environmental Mechanics and Mathematics, Springer, Berlin, 2009.

Kibler, B., Fatome, J., Finot, C., Millot, G., Dias, F., Genty, G., Akhmediev, N., and Dudley, J.: The Peregrine soliton in nonlinear fibre optics, Nat. Phys., 6, 790-795, 2010.

Kristiansen, O., Fructus, D., Clamond, D., and Grue, J.: Simulations of crescent water wave patterns on finite depth, Phys. Fluids, 17, 064101, doi:10.1063/1.1920351, 2005.

Lo, E. Y. and Mei, C. C.: Slow evolution of nonlinear deep-water waves in 2 horizontal directions - a numerical study, Wave Motion, 9, 245-259, 1987.

McLean, J. W.: Instabilities of finite-amplitude gravity waves on water of finite depth, J. Fluid Mech., 114, 331-341, 1982.

Mori, N. and Yasuda, T.: Effects of high-order nonlinear interactions on unidirectional wave trains, Ocean Eng., 29, 1233-1245, 2002

Onorato, M., Proment, D., Clauss, G., and Klein, M.: Rogue Waves: From Nonlinear Schrödinger Breather Solutions to Sea-Keeping Test, PloS one, 8, e54629, doi:10.1371/journal.pone.0054629, 2013a.

Onorato, M., Residori, S., Bortolozzo, U., Montina, A., and Arecchi, F. T.: Rogue waves and their generating mechanisms in different physical contexts, Physics Reports, 528, 47-89, $2013 \mathrm{~b}$.

Osborne, A. R.: Nonlinear Ocean Waves and the Inverse Scattering Transform, International Geophysics Series, Elsevier, San Diego, 2010.

Osborne, A. R., Onorato, M., and Serio, M.: The nonlinear dynamics of rogue waves and holes in deep-water gravity wave train, Phys. Lett. A, 275, 386-393, 2000.

Ribal, A., Babanin, A. V., Young, I., Toffoli, A., and Stiassnie, M.: Recurrent solutions of the Alber equation initialized by Joint North Sea Wave Project spectra, J. Fluid Mech., 719, 314-344, 2013.

Slunyaev, A., Kharif, C., Pelinovsky, E., and Talipova, T.: Nonlinear wave focusing on water of finite depth, Physica D, 173, 77-96, 2002.

Solli, D. R., Ropers, C., Koonath, P., and Jalali, B.: Optical rogue waves, Nature, 450, 1054-1057, 2007.

Su, M.-Y. and Green, A. W.: Wave Breaking and Nonlinear Instability Coupling, in: The Ocean Surface, edited by: Toba, Y. and Mitsuyasu, H., 31-38, Springer Netherlands, doi:10.1007/97894-015-7717-5_4, 1985.
Tanaka, M.: A method of studying nonlinear random field of surface gravity waves by direct numerical simulations, Fluid Dynam. Res.h, 28, 41-60, 2001a.

Tanaka, M.: Verification of Hasselmann's energy transfer among surface gravity waves by direct numerical simulations of primitive equations, J. Fluid Mech., 444, 199-221, 2001 b.

Toffoli, A., Bitner-Gregersen, E., Onorato, M., and Babanin, A. V.: Wave crest and trough distributions in a broad-banded directional wave field, Ocean Eng., 35, 1784-1792, 2008a.

Toffoli, A., Onorato, M., Bitner-Gregersen, E., Osborne, A. R., and Babanin, A. V.: Surface gravity waves from direct numerical simulations of the Euler equations: a comparison with second-order theory, Ocean Eng., 35, 367-379, 2008 b.

Toffoli, A., Benoit, M., Onorato, M., and Bitner-Gregersen, E. M.: The effect of third-order nonlinearity on statistical properties of random directional waves in finite depth, Nonlin. Processes Geophys., 16, 131-139, doi:10.5194/npg-16-131-2009, 2009.

Toffoli, A., Gramstad, O., Trulsen, K., Monbaliu, J., BitnerGregersen, E. M., and Onorato, M.: Evolution of weakly nonlinear random directional waves: laboratory experiments and numerical simulations, J. Fluid Mech., 664, 313-336, 2010a.

Toffoli, A., Onorato, M., Bitner-Gregersen, E. M., and Monbaliu, J.: Development of a bimodal structure in ocean wave spectra, J. Geophys. Res., 115, C03006, doi:10.1029/2009JC005495, $2010 b$.

Toffoli, A., Fernandez, L., Monbaliu, J., Benoit, M., GagnaireRenou, E., Lefevre, J. M., Cavaleri, L., Proment, C., Pakozdi, C., Stansberg, C. T., Waseda, T., and Onorato, M.: Experimental evidence of the modulation of a plane wave to oblique perturbations and generation of rogue waves in finite water depth, Phys. Fluids., 25, 091701, doi:10.1063/1.4821810, 2013.

Trulsen, K. and Dysthe, K. B.: A modified nonlinear Schrödinger equation for broader bandwidth gravity waves on deep water, Wave Motion, 24, 281-289, 1996.

Trulsen, K., Stansberg, C. T., and Velarde, M. G.: Laboratory evidence of three-dimensional frequency downshift of waves in a long tank, Phys. Fluids, 11, 235, doi:10.1063/1.869915, 1999.

Tulin, M. P. and Waseda, T.: Laboratory observation of wave group evolution, including breaking effects, J. Fluid Mech., 378, 197232, 1999.

West, B. J., Brueckner, K. A., Jand, R. S., Milder, D. M., and Milton, R. L.: A new method for surface hydrodynamics, J. Geophys Res., 92, 11803-11824, 1987.

Whitham, G.: Linear and Nonlinear Waves, Wiley Interscience, New York, 1974.

Xiao, W., Liu, Y., Wu, G., and Yue, D. K. P.: Rogue wave occurrence and dynamics by direct simulations of nonlinear wave-field evolution, J. Fluid Mech., 720, 357-392, 2013.

Yuen, H. C. and Lake, B. M.: Nonlinear dynamics of deep-water gravity waves, Adv. Appl. Mech., 22, 20-228, 1982.

Zakharov, V.: Stability of period waves of finite amplitude on surface of a deep fluid, J. Appl. Mech. Tech. Phys., 9, 190-194, 1968.

Zakharov, V. and Ostrovsky, L.: Modulation instability: The beginning, Physica D: Nonlinear Phenomena, 238, 540-548, 2009. 